\title{
Investigation and calculation of the durability of sideflexing polymer chains
}

\author{
Dr.-Ing. P. AUERbach \\ Prof. Dr.-Ing. K. NeNDEL \\ University of Technology Chemnitz, Professorship of Conveying Engineering
}

The article describes the results of fatigue tests with sideflexing polymer chains conducted on a dynamic testing machine and in testing conveyors. A new approach is suggested, that allows a calculatory estimation of the fatigue life of these chains. Finally a calculation software is presented, that has been developed based on the test results and the new equations.

\section{Introduction}

Sideflexing polymer chains for the transport of light good pieces are subjected to a number of mechanical loads, that have been described more detailed in the previously published article [Auerbach06] "Investigation of the loads and the development of tensile forces in sideflexing sliderail chains". As a result of these examinations, the (timevariant) chain tensile force was identified as the main stress factor. Furthermore, new equations for a more exact calculation of tensile forces in horizontal and vertical curves with sliding friction have been developed.

At the dimensioning of sliderail chain conveyor systems it will be checked whether the calculated chain tensile force is smaller than the feasible tensile force as denoted by the producer. An exact design is only possible when the calculated as well as the feasible tensile force are appropriate. So far a standardization and a definition respectively, of that feasible chain tensile force is missing so that every producer uses a different approach to determine this value. Therefore, the data of different producers cannot be compared with each other directly. Differing durabilities of chains are resulting that cannont be estimated arithmetically up to now.

Prerequisite for an improved dimensioning is a detailed investigation of the strength properties of sideflexing plastic chains under dynamic loads. The assumed effect of the conveyor layout onto the durability [Auerbach06] needs to be examined, which has not been done until now. The article describes the results of such researches on sideflexing finger chains with $65 \mathrm{~mm}$ plate width, consisting of three different kinds of plastic. Furthermore, an approach is presented, that for the first time allows a calculation of the chain durability. The article ends with the description of a software, that has been developed to make the dimensioning of sliderail conveyor systems based on the new approach easier.

\section{Examination of durability}

\subsection{Test methods}

At the examination of the fatigue strength of sliderail chains two competing requirements have to be weighed out against each other:

1. Examination under the most possible realistic load conditions to achieve values which can be applied on real conveyors.

2. Reduction of the generally very long testing time through large simplification of load application and time response.

If the real stresses of sliderail chains are simplified and understood as uniaxial, tumescent load, a dynamic testing machine can be used for the tests. This makes relatively high stress frequencies possible which are primarily limited through the interior damping and heating of the plastic. With that, comparatively short testing times are possible. The downside of this approach is that the results will most likely not be directly applicable onto real conveyors. 
The influence of the additional stress, especially in narrow horizontal curves on the durability, cannot be examined with the testing machine. Appropriate test conveyors were used to verify the durability under these stress conditions. Due to the necessary chain length and the limited velocity a chain circulation (that means load cycle) takes relatively long, so that this kind of testing is very time-consuming.

With both configurations experiments have been accomplished to ascertain in how far the results are matching. It was also necessary to ascertain whether a convenient combination of both systems/procedures makes it possible to achieve reliable values with a reduced expenditure of time.

\subsection{Investigations with a dynamic testing machine}

As it is recommended in the German standard DIN $8153 \mathrm{~T} 1$ for steel chains, five chain links have been gimbal-mounted in the testing machine to avoid bending stress (Figure 1).

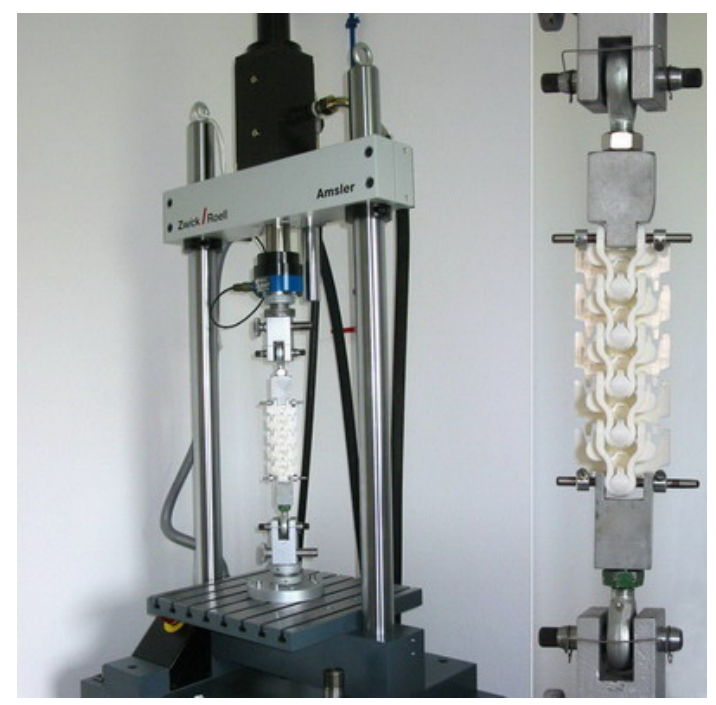

Fig. 1: Dynamic testing machine and fixation of the tested chains

Sideflexing finger chains with a plate width of $65 \mathrm{~mm}$, consisting of the three following kinds of materials have been tested:

1. Delrin ${ }^{\circledR} 500 \mathrm{Al}$ (polyacetal homo-polymer)

2. Hostaform ${ }^{\circledR}$ LW90EWX (polyacetal co-polymer)

3. Celane ${ }^{\circledR} 2002-2($ PBT)

For this the breaking load needed to be acquired first. Additionally, the amplitudes of the tumescent load for the long-term test were defined proportional to it (thirty, forty and fifty percent). Each material was tested three times at each stress level to be able to estimate the spread of the values. Because of the limited testing capacity the time-consuming examinations with 30 percent of the breaking load have been accomplished only twice and had to be cancelled at $10^{6}$ load cycles. The chosen stress frequency of two $\mathrm{Hz}$ has proven as a good compromise [Crawford 98] between an acceptable testing time and the prevention of thermal failure of the sample. Figure 2 shows the results by means of a Woehler-graph with logarithmic application of the average number of load cycles with defined amplitude of the testing load.

The dashed sections in the direction of higher load cycles are based on a linear extrapolation of the prior course. Thereby, it is assumed that no distinct fatigue strength is existing as it is the case with many plastics [Hellerich04, S. 343]. The comparison of the three materials point out that the static tensile strength does not allow a solid evaluation of the strength behaviour under dynamic stress. Thus, the Delrin has by far the highest static tensile strength but performs worse to the fatigue strength. The 


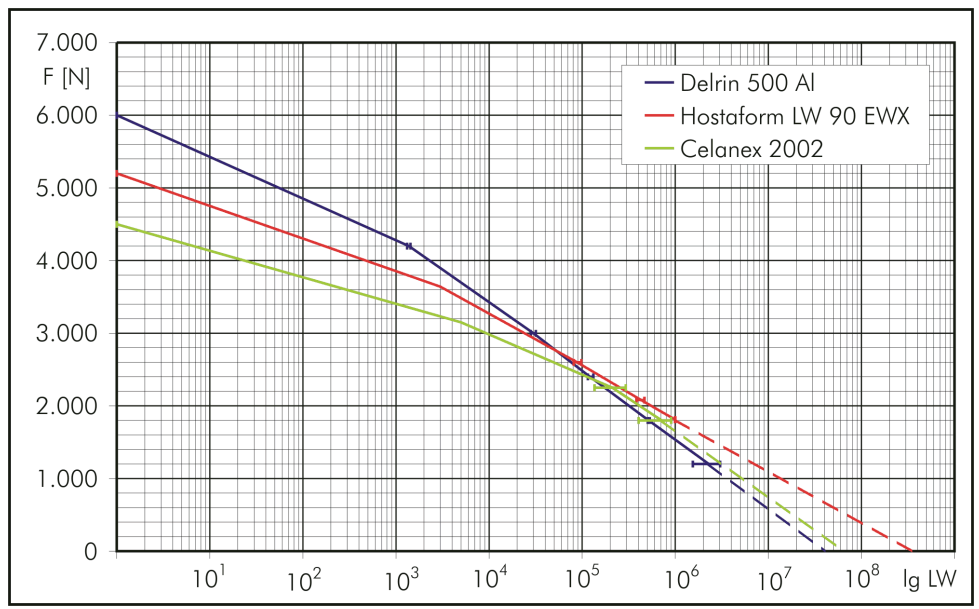

Fig. 2: Woehler-graph of the tested materials

two other materials are showing a smaller drop in strength and are achieving better dynamic strength despite smaller static tensile strength. Celanex (PBT) shows with 30percent a stronger spreading of the load cycles as the two Polyacetal-materials with about 10percent.

\subsection{Investigations on test conveyors}

For comparison to the dynamic testing machine, the durability of chains has been examined on test conveyors as well. Those consisted of a supporting frame, drive unit, deflexion unit as well as various elements for conveyor layout (straight sections, curve wheels, sliding bends), to examine their influence on the durability (Figure 3).

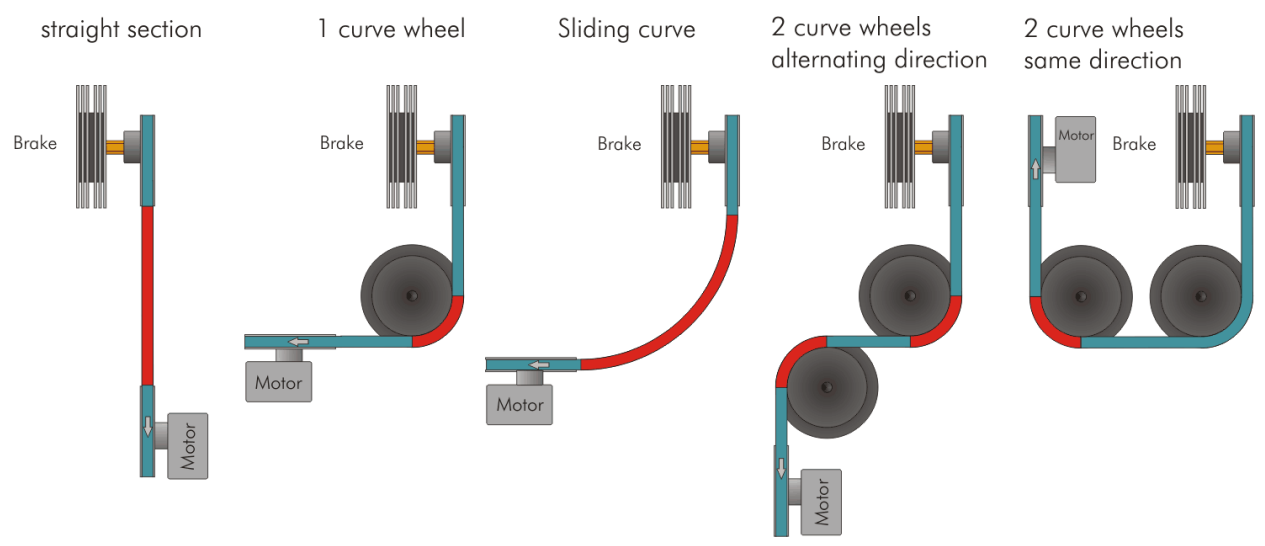

Fig. 3: Examined conveyor layouts

The curve wheels had a nominal diameter of $300 \mathrm{~mm}$, the sliding bend a medium radius of $700 \mathrm{~mm}$ and the length of the straight section was $1000 \mathrm{~mm}$. The chain stress has been realised through the deceleration (preloading) of the chain with the help of a direction change which is coupled to a magnetic powder break. The appropriate factor of proportionality was acquired through measurements of the chain tensile force at various braking torques and applied voltage, respectively. With that the wanted tensile force could be triggered through the setting up of a certain voltage. During the whole testing time the braking force has been regularly supervised with a torque support. Figure 4 shows the assembly of such a testing conveyor.

The shown layouts were examined with a maximum chain tensile force of $1000 \mathrm{~N}$. To describe the drop of strength of a chain as a Woehler-curve the layout with a curve wheel was tested with $600 \mathrm{~N}$ and $850 \mathrm{~N}$, additionally. To reduce the testing time chain links of the three materials were 


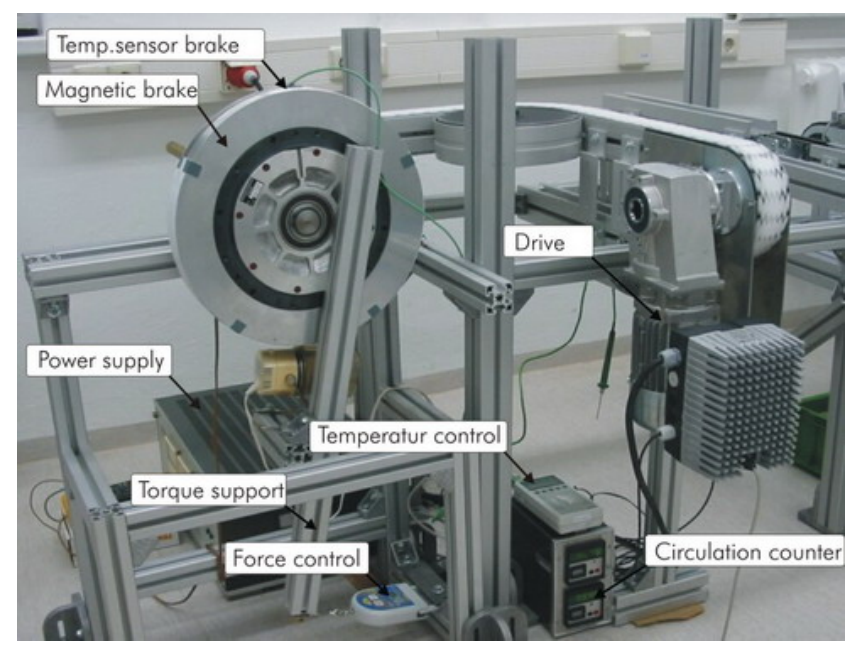

Fig. 4: Assembly of a testing conveyor

put together to one chain. The number of circulations until a link broke was recorded. The defect link was exchanged and the test continued until five links of each material broke. Figure 5 shows the average value of the recorded circulation numbers.

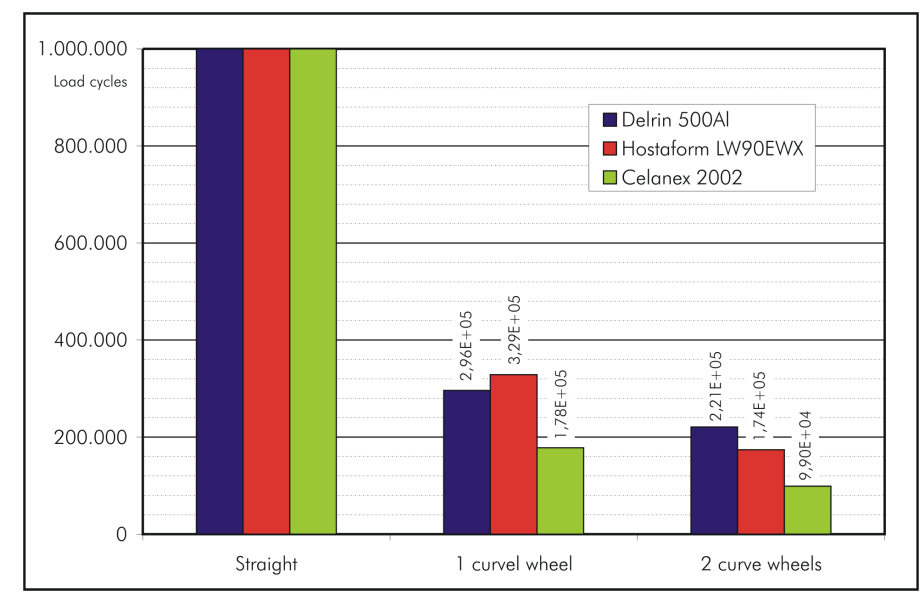

Fig. 5: Average circulation numbers until breakage

To limit the testing time, the testing of the straight section was cut out after $10^{6}$ circulations. Since the chains are loaded similarly in straight sections and testing machine, the number of cycles till fatique should be roughly equal. All materials would have endured more than $10^{7}$ cycles at $1000 \mathrm{~N}$ in the testing machine. Figure 5 reveals that the curve wheel reduces the durability of the chain by a large amount compared to the straight section.

If one chooses the $10^{6}$ load cycles of the straight section as the reference value, the curve wheel achieves only between 18\% (Celanex) and 29\% (Delrin) of it. If the layout includes two curve wheels the number of tolerated circulations is further declining to ca. 10 to $17 \%$ of the value achieved in straight sections. This corresponds to the conclusion drawn from the stress analysis. Practically, curves cause an additional load change with multiaxial tension. The more curves a section has the more frequently happens this superimposed load change - the tolerated number of circulations is reduced by that effect. The range of the fatique load cycles is similar to the range of the pulser tests. The durability of the chains made of Celane ${ }^{\circledR}$ spreads with ca. $30 \%$ more than the ones made of Delrin ${ }^{\circledR}$ - or Hostaform ${ }^{\circledR}$, which spread only with $10 \%$.

The big friction force and the strong heating of the slide bar and chain made it impossible to investigate the fatique cycles of the conveyor with a horizontal sliding curve. Already in a pre- test, the chosen 
amplitude of $500 \mathrm{~N}$ melted the chain in the contact area of the inner part to the sliderail. To prevent a quick destruction of the chain through excessive wear and material removal the test had to be stopped. Apparently, the thermical stress of chain and slide bar when using sliding curves restricts the tolerable tensile force and durability of the chain.

\subsection{Comparison of testing methods}

As mentioned earlier, the durability test on test conveyors needs much more time and test effort as on the material testing machine. By comparison of the results from both procedures a correlation has been searched which allows a reduction of this effort but still provides practicable values (Figure 6).

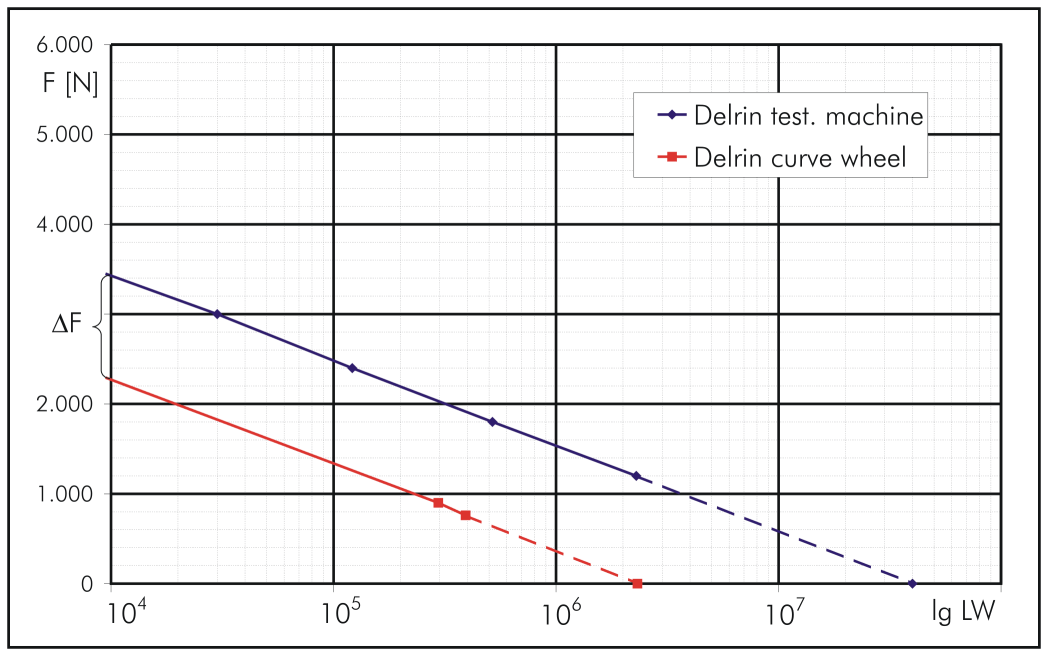

Fig. 6: Comparison of the Woehler-lines of pulser and test conveyor

The lines of the test conveyor are based on the medium numbers of circulations till breakage at three load levels. Figure 6 shows by the example of Delrin ${ }^{\circledR}$ that the chain while tested at the test conveyor tolerates clearly smaller forces. The drop in strength is similar in both cases. The other two materials show a similar correspondance. If this correlation can be acknowledged with other types of chains and material, only one test on a conveyor would remain to define the displacement $F$. Then, the Woehler-line of the test conveyor is incidental through the parallel translation of the curve $\mathrm{F}$ acquired onto the testing machine.

\section{Estimation of durability}

\subsection{Suggested approach}

The usual method of dimensioning which related to the static tensile strength could not make any statement about the durability of chains. As the results of the tests are showing, different kinds of material showed a differently strong drop in dynamic strength which does not relate to the static tensile strength. In the conveyor with curves, the chains only reached a small durability.

An approach was searched which permits an estimation of that durability and with that a more exact dimensioning. Because of the displayed, manifold factors onto the stress and the durability some simplifications were necessary. The course of the tensile force will be understood as tumescent, sinusoidal load while cycling through the conveyor. In the real operation of conveyors the amount of transported cargo will not be constant but will change with the working conditions of the conveyor. To describe this differing loads mathematically, a summary of situations with similar stress is necessary. The suggestion is to distinguish between the working conditions start, idling chain, cargo transport and accumulation mode.

Every working condition is marked by a different maximum tensile force. Altogether, the stresses can be understood as multi-stage complex of loads acting on a component. The so-called Palmgren-Miner 
rule, a hypothesis of a linear damage accumulation, has achieved [Zammert85] a prevalence on the area of operational stability. This rule permits the affiliation of the durability of a multi-stage stressed component onto the Woehler-curves which have been acquired in a single level test. For this purpose, a partial damage $D_{i}$ has to be acquired for each load level, which results from the quotient of completed $\left(n_{i}\right)$ and permissible number of cycles $\left(N_{i}\right)$ :

$$
D_{i}=\frac{n_{i}}{N_{i}}
$$

The part fails, when the total damage $D$ reaches the value of one.

$$
D=\sum_{i} D_{i}=\sum_{i} \frac{n_{i}}{N_{i}}=1
$$

The number of cycles of load level $i$ can be calculated with its temporal frequency $H_{i}$ in relation to the total number of cycles $n$ :

$$
n_{i}=H_{i} \cdot n
$$

After insertion of this in (2) results:

$$
D=\sum_{i} \frac{n_{i}}{N_{i}}=\sum_{i} \frac{H_{i} \cdot n}{N_{i}}=n \cdot \sum_{i} \frac{H_{i}}{N_{i}}=1
$$

Equation 4 can be transformed to get the total number of tolerable cycles:

$$
n=\frac{1}{\sum_{i} \frac{H_{i}}{N_{i}}}
$$

Together with chain length $L_{G}$ and speed $v$ the tolerable operating time $t$ can be calculated:

$$
t=\frac{L_{G}}{v \cdot 3600} \cdot n
$$

The following example may demonstrate how this approach can be used to calculate the durability of plastic sliderail chains.

\subsection{Example calculation}

A calculation based on the described approach requires following steps:

1. Calculation of the amplitudes of the tensile force

2. Determination of the tolerable number of cycles

3. Calculation of durability

The working phases are defined by their amplitude of tensile force as well as by their relative frequency (related to the total running time). For the calculation of the tensile force a reference to the prior article and the equations has to be done.

The frequency needs to be acquired or estimated out of the technological edge conditions. For this example the values, described in table 1 will be adopted.

Out of the resistance chart of the applied chain the tolerable number of cycles has to be read off for each amplitude.

To demonstrate the use (2.4) of it, it is assumed that the conveyor contains a curve wheel. The tolerable number of cycles $N$ of the conveyor can be read off of the chart for the testing machine if the displacement $\Delta F$ will be added to the appropriate tensile force. A Delrin chain shall be used, for 
Table 1: Given data of the operating phases

\begin{tabular}{|c|c|c|c|}
\hline i & Operating phase & Frequency & Max. tensile force \\
\hline 1 & Idling chain & 0,2 & $300 \mathrm{~N}$ \\
\hline 2 & Transport & 0,5 & $500 \mathrm{~N}$ \\
\hline 3 & Accumulation & 0,2 & $650 \mathrm{~N}$ \\
\hline 4 & Start & 0,1 & $700 \mathrm{~N}$ \\
\hline
\end{tabular}

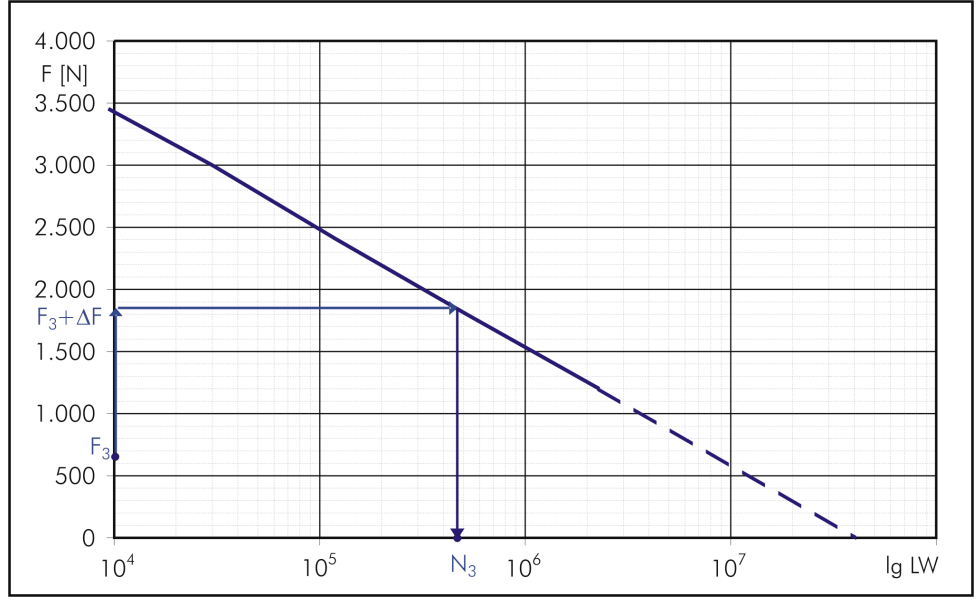

Fig. 7: Determination of tolerable cycles

which a $\Delta F$ of $1200 \mathrm{~N}$ was acquired in the tests. Figure 7 illustrates by the example of the strength amplitude for the accumulation mode $\left(F_{3}=650 \mathrm{~N}\right)$ the way the metering is happening.

If this has been done with all four amplitudes one achieves the complete dataset for the working conditions as described in chart 2 .

With the given data the total number of tolerable chain circulations can be calculated with equation 5 . After inserting all values one achieves:

$$
n=\frac{1}{\frac{0,2}{1,0 \cdot 10^{6}}+\frac{0,5}{5,2 \cdot 10^{5}}+\frac{0,2}{4,4 \cdot 10^{5}}+\frac{0,1}{3,0 \cdot 10^{5}}}=5,13 \cdot 10^{5}
$$

Considering a chain length of $10 \mathrm{~m}$ and a speed of $0,5 \mathrm{~m} / \mathrm{s}$ following tolerable running time is resulting:

$$
t=\frac{10 m}{0,5 \mathrm{~m} / \mathrm{s} \cdot 3600 \mathrm{~h} / \mathrm{s}} \cdot 5,13 \cdot 10^{5}=2^{\prime} 850 \mathrm{~h}
$$

With the described course the durability of sliderail chains until breakage can be calculated for any kind of conveyor. The following section describes possible sources of inaccuracies in the calculation.

Table 2: complete dataset of the working conditions of the example

\begin{tabular}{|c|c|c|c|c|}
\hline i & Working condition & Frequency & Max. tensile force & Number of cycles \\
\hline 1 & Idling chain & 0,2 & $300 \mathrm{~N}$ & $1,0 \cdot 10^{6}$ \\
\hline 2 & Transport & 0,5 & $500 \mathrm{~N}$ & $5,2 \cdot 10^{5}$ \\
\hline 3 & Accumulation & 0,2 & $650 \mathrm{~N}$ & $4,4 \cdot 10^{5}$ \\
\hline 4 & Start & 0,1 & $700 \mathrm{~N}$ & $3,0 \cdot 10^{5}$ \\
\hline
\end{tabular}




\subsection{Possible source of errors}

The described approach bases on some assumptions and includes values which are partly statistically distributed. Therefore, the calculated durability will also be afflicted with a some kind of uncertainty. Figure 8 shows the three main categories of possible sources of errors in an outline.

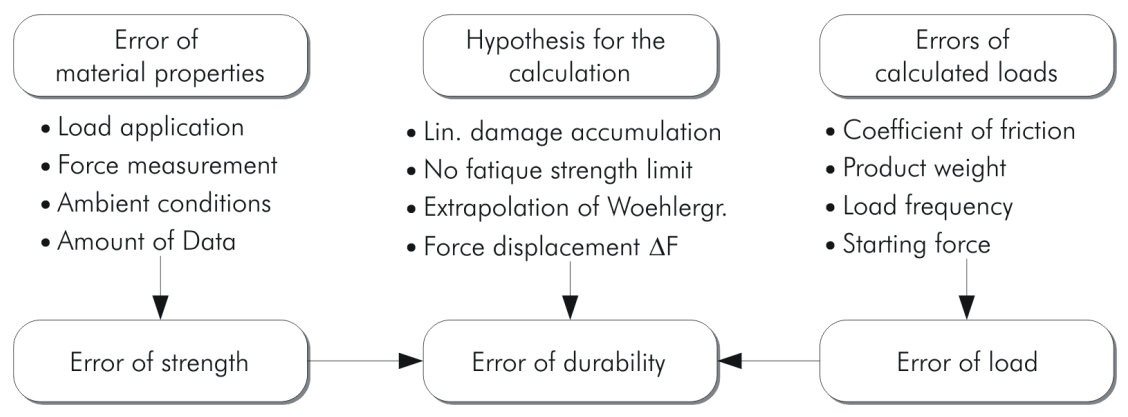

Fig. 8: Possible error sources

The first category includes errors of the experimentally acquired material characteristics (Woehlercurves) which, besides others, can be caused through tolerances of the gauge and the load application within the tests, as well as through fluctuations of the material characteristics or environmental conditions. The second category contains inaccuracy which results out of the adoption or simplification of the calculated approach. The last category depends on the accurateness of the operator. This effects not only the accuracy of the calculated chain tensile forces which requires especially exact coefficient of friction and assembly weight, but also the assumed frequencies of the working conditions which need to go conform with real cases of operation. Many of the mentioned sources of errors are not quantifiable so that no number of the overall error of durability can be given. Verifications of the Miner rule on results of experiments, as documented in the literature, show that this rule offers appropriate results on an average [Haibach02]. Admittedly, the acquired totals of damage featured quite a big spreading range, depending on material, type and order, in which the load levels were applied [Buxbaum92].

Inspite of this, for the first time the described approach permits at least a rough estimation of the durability of plastic sliderail chains. Therewith, it is an essential progress towards the previous construction of the static tensile strength and a flat safety factor. It was not possible to present a picture showing the durability before.

\section{Calculation software}

Adequate software for computers was developed to ease the dimensioning of sliderail chain conveyor systems as the described approach had introduced. It was essential to focus on a user-friendly design of the functions and the surface of the program. Necessary characteristic values for the calculation of drives, chains, and slide bars were deposit in a databank to reduce the amount of the necessary user input. All other inputs occur in four concise steps. Figure 9 shows exemplary the design of the dialogue for the configuration of the conveyor which is supported by a schematic preview of the traffic routing.

The results (tensile force, durability, drive power) are displayed in the last step and a printout of the calculation is provided (Figure 10).

At the moment the program exists in the German and English language. Translations into more languages require only small effort due to the realised separation of labels and source code. Database and program are designed so that the range of goods of different producers can be integrated with only little changes. Appropriately adapted versions of the program are in the testing period with different providers of sliderail chain conveyor systems. 


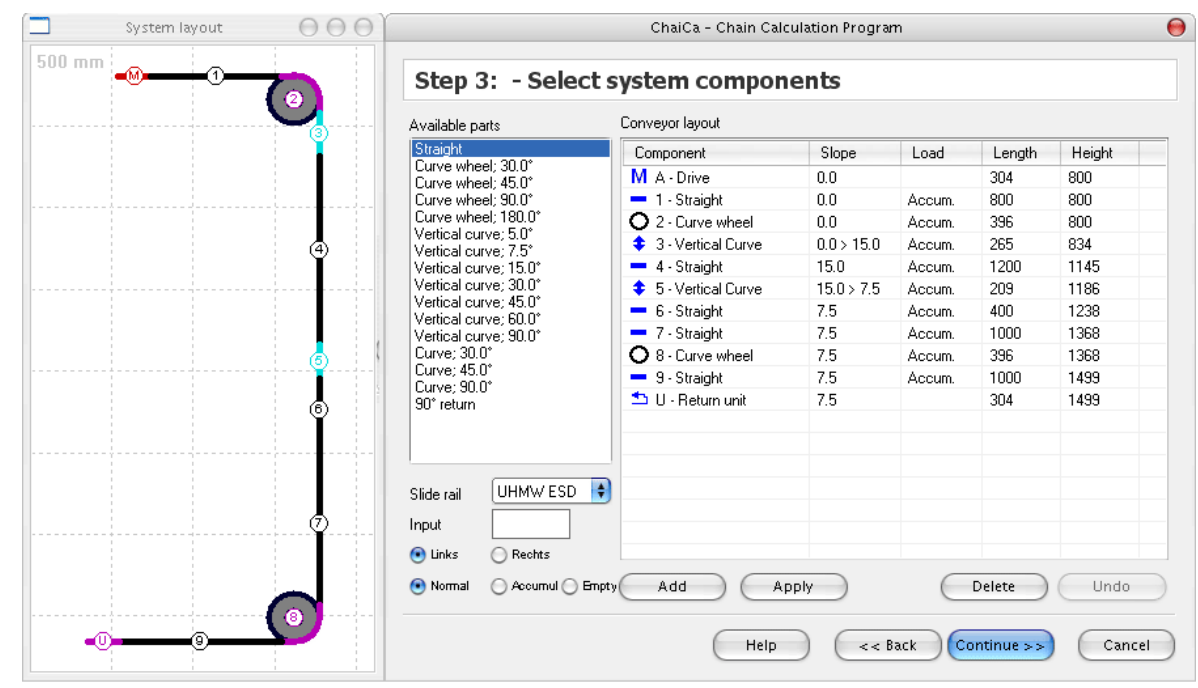

Fig. 9: Dialogue for determination of the conveyor layout

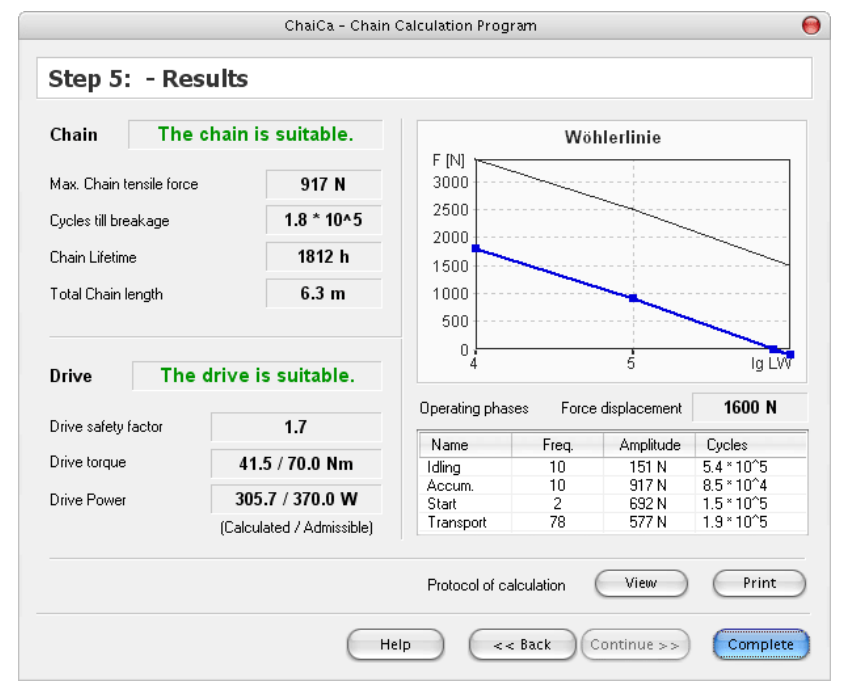

Fig. 10: Display of the results

\section{Conclusion and outlook}

The results show that the durability of sideflexing plastic sliderail chains is not only dependent on the maximum cargo load or the chain tensile force but also on the section layout of the conveyor. When applying sliding curves in a conveyor the thermal strength of chain and slide bar is appointing the limit of use. The stress needs to be limited so much that partial melting or excessive wear can be avoided. In this case the chain has to be replaced because it has reached a certain wear limit, even before a fatique breakage occurs.

In cases where curve wheels are used which eliminate friction to a large extend but stress the chain links more due to the superimposed bending, the durability of a chain is limited by its fatigue strength. The reduction of the durability due to the curve wheels compared to straight sections at the same maximum tensile force has been displayed. Beyond that, an approach has been proposed which allows an estimation of the durability based on the aquired strength lines. It was mentiond that a lot of the used parameters are statistically distributed and hence, inevitably lead to certain precariousness or spreading of the calculated durability. Nevertheless, this approach is a clear progress compared to the previous design method based solely on the static breakage force, because for the first time it allows for an approximate appraisal of the expected durability. 
Further investigations within this field could deal with the following topics:

- Investigations of the fatigue strength on other types of chains and material verification of the connection between the results of testing machine and test conveyor

- more exact analysis of the thermal limits of use, creation of the dimensioning basis for friction and heating

- analysis of the abrasion at plates and linking elements as well as on the increase of the chain pitch, arithmetical description of the interrelationship

The results of this investigations could present useful animations for constructive or material optimizations which allow an increase of strength, durability and friction resistance of the sliderail chains.

\section{Symbols}

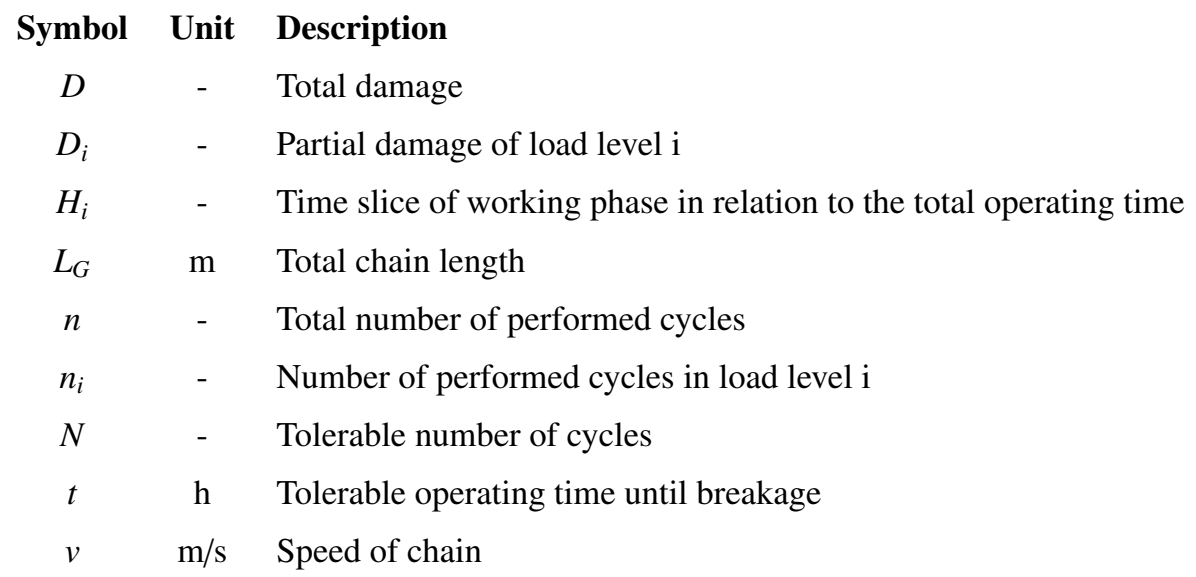

\section{References}

[Auerbach06] Investigation of the loads and the rise of tensile forces in sideflexing sliderail chains. In: Logistics Journal (2006)

[Buxbaum92] Buxbaum, Otto: Betriebsfestigkeit: Sichere und wirtschaftliche Bemessung schwingbruchgefährdeter Bauteile. 2nd edition. Berlin : Verlag Stahleisen, 1992

[Crawford98] Crawford, R.J.: Plastics Engineering. Third Edition. Oxford : ButterworthHeinemann, 1998.

[Haibach02] Haibach, Erwin: Betriebsfestigkeit: Verfahren und Daten zur Bauteilberechnung. 2nd edition. Berlin, Heidelberg : Springer, 2002.

[Hellerich04] Hellerich, W.; Harsch, G.; Haenle, S.: Werkstoff-Führer Kunststoffe. 9th edition. München, Wien : Carl Hanser Verlag, 2004.

[Zammert85] Zammert, W.U.: Betriebsfestigkeitsberechnung. Braunschweig : Vieweg, 1985. 